\title{
Efeitos Nutricionais e Mecanismos Bioquímicos na Doença Hepática Gordurosa Não Alcoólica
}

\author{
Nutritional Effects and Biochemical Mechanisms in Non- \\ alcoholic Fatty Liver Disease
}

Wilson Malagó-Jr', Angélica de Moraes Manço Rubiatti², Cristina Ferro Correa Toniolo², Valéria Cristina Schneider ${ }^{2}$

${ }^{7}$ Centro Universitário Central Paulista - UNICEP

${ }^{2}$ Professora do Curso de Nutrição do Centro Universitário Central Paulista - UNICEP

E-mail para contato: Wilson Malagó-Jr - malago.jr@gmail.com

\section{Resumo}

A Doença Hepática Gordurosa Não Alcoólica (DHGNA) tem ganhado proporções alarmantes, afetando de 25 a 30\% da população mundial. Sua ocorrência está associada ao excesso de peso, resistência à insulina e síndrome metabólica. Ela é uma doença silenciosa que evolui do acúmulo de gordura no fígado para a hepatite, fibrose, cirrose e carcinoma. A morbimortalidade associada à DHGNA tem aumentado significativamente, junto com o crescimento do excesso de peso na população. Ainda não há um marcador específico para o diagnóstico, e nem um tratamento farmacológico para a doença. Portanto, a prevenção e o tratamento se baseiam na mudança do estilo de vida, com a adoção de hábitos alimentares saudáveis, que devem necessariamente levar à perda de peso, para a melhora do quadro. O objetivo deste trabalho foi pesquisar e sintetizar informações científicas atualizadas acerca da DHGNA. As buscas, com os descritores utilizados, foram direcionadas às influências dietéticas no desenvolvimento, prevenção e tratamento da DHGNA e aos mecanismos bioquímicos e metabólicos envolvidos com a doença. Foram disponibilizadas informações que ajudam a entender a dietética e a bioquímica no da DHGNA, além da importância da doença em termos de saúde pública. Os temas reportados ajudam a ambientar o profissional nutricionista no cenário atual da educação alimentar e nutricional, voltadas para a prevenção e tratamento da doença.

Palavras-chave: Esteatose Hepática não Alcoólica. Terapia Nutricional. Metabolismo.

\begin{abstract}
The Non-alcoholic Fatty Liver Disease (NAFLD) has gained alarming proportions, affecting about 25-30\% of the world's population. The occurrence of NAFLD is associated with overweight, insulin resistance, and metabolic syndrome. It is a silent disease that evolves from an initial fat liver accumulation to the steatosis, hepatitis, fibrosis, cirrhosis, and carcinoma. Morbimortality associated with NAFLD has increased significantly, along with the growth of overweight in the population. There is still neither a specific marker for the diagnosis nor a pharmacological treatment for the disease. Therefore, both the prevention and treatment are based on a change in lifestyle, with the adoption of healthy eating habits that must lead to weight loss, improving the health condition. The objective of this
\end{abstract}


review was to search and synthesize updated scientific information about the DHGNA. The search was directed to dietary influences in the development, prevention, and treatment of NAFLD and to the biochemical and metabolic mechanisms involved with the disease. By doing that we could provide information to help understanding the DHGNA's diet and biochemistry, as well as the importance of the disease on the light of public health. The issues reported help to enhance the nutritionist in the current scenario of food and nutrition education, aimed at the prevention and treatment of the NAFLD.

Keywords: Non-alcoholic Hepatic Steatosis. Nutritional Therapy. Metabolism.

\section{INTRODUÇÃO}

As doenças hepáticas crônicas (DHC) representam um dos maiores problemas de saúde pública. Elas são majoritariamente representadas pela doença hepática alcoólica (DHA), pela doença hepática gordurosa não alcoólica (DHGNA) e pelas hepatites B e C. Estimativas de 2014 apontaram que cerca de 850 milhões de pessoas no mundo são portadoras de DHC. As DHCs são doenças silenciosas, sub diagnosticadas, que têm causas distintas e que podem ocorrer associadamente ${ }^{1}$. Uma das formas mais comuns de doença hepática é a doença hepática gordurosa não alcoólica (DHGNA)².

A DHGNA tem crescido no mundo em associação com maus hábitos alimentares e estilo de vida sedentário, junto com a epidemia de obesidade ${ }^{3}$. Esta doença afeta de 25 a 30\% da população mundial 4 . A ocorrência da DHGNA está associada à obesidade, resistência à insulina e síndrome metabólica, sendo inclusive considerada a manifestação hepática dessa síndrome. Essas doenças compartilham fatores de risco: obesidade, diabetes tipo 2, dislipidemia e resistência à insulina ${ }^{4,5}$.

A esteatose hepática se caracteriza pelo acúmulo de gordura no fígado, principalmente por triglicerídeos, que excede $5 \%$ do peso do órgão. A DHGNA é definida como uma esteatose hepática em que o acúmulo de gordura não está associado a hepatites virais, consumo de álcool, problemas autoimunes e doenças metabólicas ${ }^{4,6}$. Ela se caracteriza pelo acúmulo de gordura e pelo desenvolvimento de inflamação no fígado ${ }^{6}$. A preocupação com a DHGNA, além de ter um risco cardiovascular associado, reside no fato de que ela é uma doença silenciosa, que evolui do simples acúmulo de gordura, para a esteatose associada com hepatite (inflamação) e fibrose, até o desenvolvimento de cirrose hepática e/ou carcinoma hepatocelular ${ }^{3,7}$. O carcinoma hepatocelular pode se desenvolver também na 
ausência de cirrose, em pacientes ainda com quadro de fibrose. Isso acontece em cerca de $40 \%$ dos $\operatorname{casos}^{8}$.

Apesar de os pacientes com DHGNA terem um risco de morte associada ao fígado 10 vezes maior, em relação às pessoas não portadoras, a causa mais comum de morte entre eles é decorrente do risco cardiovascular associado, que se encontra duas vezes aumentado quando comparado aos não portadores de DHGNA ${ }^{4,5}$. Entretanto, as hepatites crônicas virais vêm sendo bem controladas com uso dos antivirais de ação direta e, por outro lado, a cirrose e o carcinoma hepatocelular associados à DHGNA vêm aumentando ${ }^{9,10}$. Essas complicações da DHGNA, apesar de evoluírem atualmente em somente $2,5 \%$ dos casos, já representam a segunda causa de transplante de fígado nos Estados Unidos, atrás apenas da hepatite $\mathrm{C}^{4}$. Tem sido previsto que nos próximos 20 anos a DHGNA se tornará a principal causa de morbimortalidade associada ao fígado, liderando as indicações de transplante desse órgão ${ }^{11}$.

A condição chave para a progressão da DGHNA é a inflamação do fígado ${ }^{12}$. Com o acúmulo de triglicerídeos no citoplasma, ocorre a destruição dos hepatócitos, a resposta imune e a inflamação. Isso induz a progressão para fibrose, cirrose hepática e carcinoma hepatocelular ${ }^{13}$. Por isso, essa inflamação deve ser detectada e tratada o quanto antes. Se a inflamação for anulada, a fibrogênese cessa e ocorre uma fibrólise natural. Com isso, até a cirrose pode regredir a exemplo dos tratamentos de hepatite B e C com antivirais. Portanto, a inflamação deve ser o alvo de terapia para a DHGNA $^{14,15}$. Entretanto, a DHGNA é assintomática, com sinais clínicos tardios que ocorrem a partir do quadro de fibrose acentuada. Por isso, o diagnóstico precoce ocorre normalmente por conta de achados incidentais de níveis alterados de enzimas que atuam no fígado, associados a exames de imagem que sugerem a esteatose. A confirmação da inflamação se dá por meio de biópsia ${ }^{16}$.

Há alguma associação dos níveis de enzimas, como a alanina aminotransferase, como marcador de inflamação de hepatócitos, para o diagnóstico da evolução da DHGNA. No entanto, no geral, os níveis desta e de outras enzimas monitoradas nos exames, e que atuam no fígado, não obedecem a um padrão confiável que permite diagnosticar a presença ou ausência da doença. Esses exames apenas levantam suspeitas acerca da DHGNA. O desconhecimento da complexidade molecular da patogênese e da progressividade da DHGNA explica o fato de não haver ainda um marcador específico dedicado para a deteç̧ão da doença, bem como, explica a 
inexistência de um tratamento farmacológico específico. Consequentemente, também não há políticas públicas específicas, em nenhum país do mundo, voltadas para o diagnóstico e tratamento da DHGNA ${ }^{15}$.

Deste modo, um melhor entendimento dos mecanismos moleculares envolvidos na DHGNA, no acúmulo de gordura e no desenvolvimento de inflamação e fibrose, é crucial para a viabilidade do desenvolvimento de testes específicos e de uma terapia farmacológica ${ }^{5,17}$. Com a inexistência de drogas para o tratamento da DHGNA, a recomendação é a mudança do estilo de vida, incluindo a adoção de hábitos alimentares saudáveis e a prática de exercícios físicos, que devem necessariamente levar à perda de peso. Estas medidas reduzem a inflamação hepática e os danos celulares da DHGNA, além de diminuir o risco cardiovascular associado. Alguns profissionais associam ao tratamento a prescrição de drogas usadas para dislipidemia e resistência à insulina ${ }^{17}$.

Neste contexto, os profissionais de saúde estão diante do desafio de implementar urgentemente ações que incluam prevenção, diagnóstico precoce, educação e tratamento, para o combate da DHGNA ${ }^{1}$. Com isso, o profissional nutricionista se faz necessário para a educação alimentar e nutricional, na orientação dos pacientes, para a prevenção e o tratamento da DHGNA. O presente trabalho objetivou trazer esclarecimentos a cerca da relação entre a dietética e a bioquímica com o desenvolvimento da DHGNA, no intuito de ambientar os profissionais nutricionistas com a problemática da doença.

\section{MÉTODO}

Foi realizada uma revisão narrativa a fim de reunir e disponibilizar informações a cerca da DHGNA, para trazer um panorama atual do conhecimento científico sobre a fisiopatologia, prevenção e tratamento da doença. O foco das buscas foi direcionado às relações entre a dietética e a bioquímica com a DHGNA. Entretanto, foram reunidas também algumas informações de saúde pública e sobre a importância clínica da doença. Foram acessados artigos científicos publicados em revistas indexadas, documentos e livros sobre o tema. As bases de dados acessadas foram PubMed, Scielo, Science Direct, Projeto Diretrizes Clínicas (ANS-AMB), Portal Saúde Baseada em Evidências (PSBE) e Biblioteca Virtual em Saúde (BVS). Além da plataforma de busca Google Acadêmico e websites, que reúnem publicações de instituições civis e governamentais da saúde, e opiniões de profissionais. A revisão foi realizada entre 13 de setembro de 2018 e 11 de julho de 2019. 
Os descritores utilizados foram escolhidos a partir da lista de Descritores em Ciências da Saúde (DeCS) da Biblioteca Virtual em Saúde (BVS) ${ }^{18}$. As buscas foram efetuadas em inglês e português, por meio de combinações dos termos e de seus sinônimos a seguir: Fatty Liver - Fígado Gorduroso (Esteatose Hepática, EsteatoHepatite); Non-Alcoholic Fatty Liver Disease - Hepatopatia Gordurosa não Alcoólica (Doença Hepática Gordurosa não Alcoólica, Esteato-Hepatite não Alcoólica); Etiology - Etiologia (Causalidade, Causas, Patogênese); Metabolism - Metabolismo (Anabolismo, Catabolismo, Conceitos Metabólicos, Fenômenos Metabólicos, Processos Metabólicos); Nutrition Therapy - Terapia nutricional (Terapia Nutricional Médica, Terapia Médica Nutricional).

\section{DESENVOLVIMENTO}

\section{Particularidades da DHGNA}

A DHGNA tem se mostrado uma importante doença para a saúde pública. Ela tem crescido significativamente, principalmente relacionada aos maus hábitos alimentares ${ }^{3,4}$. No entanto, em casos menos comuns, a DHGNA também pode estar associada à rápida perda de peso, ao uso de medicamentos hepatotóxicos, ao uso de esteroides anabolizantes e à exposição a toxinas, particularmente as aflatoxinas ${ }^{19}$, 20,21 .

O excesso de peso está associado à DHGNA. Entretanto, existem alguns casos, bem menos frequentes, de pacientes eutróficos que apresentam a doença, em geral, mais branda e com melhores chances de recuperação, em relação aos pacientes com sobrepeso ou obesidade. Estes pacientes, apesar de eutróficos, normalmente também apresentam resistência à insulina, dislipidemia, diabetes tipo 2 e risco cardiovascular. Nestes casos a ocorrência de um leve excesso de gordura visceral abdominal pode estar associada ao desenvolvimento da DHGNA ${ }^{22}$.

\section{Agravamentos}

A inflamação do fígado é responsável pela progressão do quadro de DGHNA para a fibrose. A fibrose é, portanto, decorrente da morte celular por necrose, provocada pelo acúmulo de ácidos graxos e pelo estresse oxidativo ${ }^{12,13}$ (Figura 1).

Há dois tipos de morte celular. Uma programada, chamada de apoptose, que ocorre de forma organizada e que não gera reação inflamatória. Outra induzida por fatores agressivos, como temperatura alta, pHs extremos, infecção viral, acúmulo de ácidos graxos, entre outros. Essa é denominada necrose e provoca reação inflamatória. Na 
apoptose a morte celular está associada a situações fisiológicas. As células são condensadas, concentram as organelas de modo organizado e acabam sofrendo fagocitose por macrófagos. Na necrose, a morte celular está associada a uma situação patológica. As células ganham volume, se desorganizam e têm as membranas das organelas rompidas, por conta do agente agressor. Deste modo, as células se rompem induzindo lesões inflamatórias. Os ácidos graxos em concentrações supra-fisiológicas, como nos casos de hepatócitos em quadros de DGHNA, causam necrose ${ }^{23}$.

Após a necrose e a consequente lesão inflamatória, se instala um quadro de fibrose hepática que pode evoluir para cirrose e carcinoma hepatocelular (Figura 1). Ao repor e reparar o tecido lesionado por necrose ocorre a cicatrização e, por meio de repetidas lesões e cicatrizações, se instala o quadro de fibrose. A cirrose é caracteriza pela cicatrização, ou fibrose, disseminada no fígado, que leva a uma distorção do órgão. Em um quadro de fibrose grave, que caracteriza a cirrose, a estrutura do fígado é prejudicada a ponto de bloquear o fluxo sanguíneo e o fluxo da bile, e de comprometer as funções hepáticas. O carcinoma hepatocelular pode evoluir após a manifestação da fibrose ou da cirrose ${ }^{8,24}$ (Figura 1).

Figura 1 - Progressão da DHGNA. O acúmulo de gordura promove a necrose e a lesão inflamatória. O quadro evolui para fibrose hepática, cirrose e carcinoma hepatocelular.

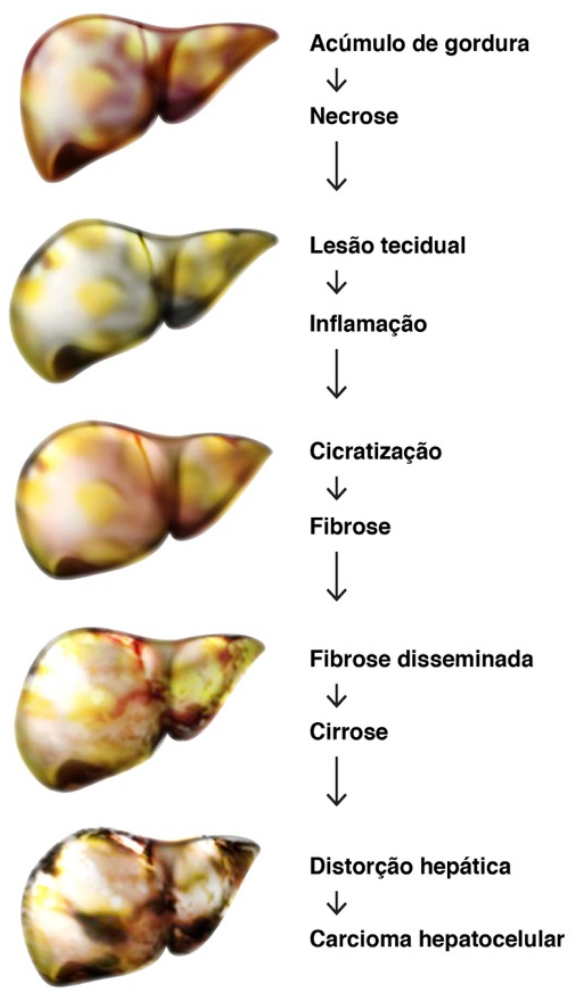




\section{Diagnóstico}

Existem vários testes baseados em marcadores que, por um motivo ou por outro, não contribuem efetivamente para o diagnóstico da DHGNA. Por exemplo: a determinação sérica da citoqueratina 18 (CK-18), que avalia o grau de necrose; o teste de tioredoxina e ferritina, que avaliam o estresse oxidativo; os testes de ácido hialurônico, laminina e inibidor de metaloproteinase (TIMP), que identificam, a presença de fibrose. Todos esses testes, além de não estarem disponíveis e difundidos na prática clínica, se prestam para identificar apenas estágios mais avançados da doença, a partir da inflamação. No entanto, nesses estágios os sinais clínicos aparecem e facilitam o diagnóstico. Há também problemas, entre os marcadores disponíveis, de apresentarem variações entre indivíduos e, portanto, não serem aplicáveis a todos ${ }^{25}$.

Outros métodos já se mostram mais úteis. A biópsia hepática é o padrão ouro para a identificação das formas da DHGNA. Mas a elevada prevalência da doença inviabiliza a realização de biópsias em todos os pacientes. O ultrassom é prático e simples, mas identifica com segurança apenas esteatoses que excedem $30 \%$ do fígado. Há também a ressonância magnética que funciona a contento para esteatoses entre 5 a 30\%, mas esbarra na inviabilidade econômica, em termos de adoção de políticas publicas em saúde. Por fim, a elastografia hepática ultrassônica, que avalia a elasticidade do tecido, é uma opção confiável e barata para identificar esteatoses. No entanto, ela identifica esteatoses menos intensas, de até $5 \%$

\section{Bioquímica e metabolismo}

Os nutrientes são recebidos e distribuídos pelo fígado. Ele é o primeiro órgão a ter acesso aos nutrientes absorvidos na digestão, além de receber também nutrientes das reservas do organismo. As quantidades e tipos de nutrientes que chegam ao fígado variam de acordo com a dieta e o intervalo entre as refeições. A exportação de nutrientes atende à demanda dos tecidos extra-hepáticos, que varia entre os órgãos e com a atividade e o estado nutricional do indivíduo ${ }^{27}$.

Com a gordura não é diferente. O fígado é o regulador central do metabolismo de lipídeos no organismo. Ele recebe os lipídeos circulantes, comanda a síntese de novos lipídeos, exporta lipídeos para tecidos não hepáticos, além de usar a energia dos lipídeos para os hepatócitos, via beta-oxidação. A esteatose hepática é consequência de um quadro em que a aquisição de lipídeos é maior do que sua 
remoção ou destinação, ou seja, nesses casos, a internalização de lipídeos e a lipogênese no hepatócito são maiores do que a oxidação e a exportação de lipídeos ${ }^{28}$. O balanço entre a aquisição e a destinação de lipídeos é regulado por quatro vias principais: aquisição de lipídeos circulantes, lipogênese, oxidação de ácidos graxos e exportação de lipídeos via VLDL-c (Figura 2). Esses processos são regulados por interações complexas entre hormônios, receptores celulares e nucleares e fatores de transcrição. Todos esses reguladores mantêm a homeostase hepática lipídica sob controle rigoroso ${ }^{29}$. Condições patológicas que causam o comprometimento de um ou mais desses componentes podem provocar a retenção de ácidos graxos nos hepatócitos, desenvolvendo a DHGNA. Entretanto, apesar de haver importantes esclarecimentos já publicados, os mecanismos moleculares responsáveis pelo acúmulo de lipídeos no fígado ainda não estão plenamente elucidados ${ }^{28}$.

O excesso de peso e a resistência à insulina favorecem um alto influxo de ácidos graxos no fígado. Em condições de jejum, após o esgotamento das reservas de glicogênio hepático, os ácidos graxos são liberados do tecido adiposo e retornam ao fígado via albumina sérica. Parte desses ácidos graxos pode ser secretada via VLDLc para tecidos extra-hepáticos, parte pode ser usada para geração de energia no hepatócito via oxidação. Em casos de DHGNA, uma parte tende a ser acumulada no hepatócito. Por outro lado, em uma situação pós-prandial, em paciente com síndrome metabólica, uma grande captação de ácidos graxos pelo fígado é atribuída a uma grande liberação de lipídeos, a partir de uma massa expandida de tecido adiposo, como consequência da resistência à insulina. De fato, a resistência periférica à insulina favorece a lipólise da gordura e um maior influxo de ácidos graxos no fígado. Neste caso, a insulina, com a sua sinalização comprometida, deixa de promover a supressão de lipólise nos adipócitos, pela incapacidade de reduzir a atividade das lipases (Figura 2). Desta forma, mesmo com a disponibilidade de energia oferecida por carboidratos, em uma situação de glicemia, ocorre a liberação de ácidos graxos circulantes, que deveria estar inibida. Nesse contexto, quanto maior a massa gorda, maior a contribuição para o aumento de liberação de ácidos graxos do tecido adiposo e sua captação pelo fígado. Por isso, a DHGNA é associada ao excesso de peso e à resistência à insulina ${ }^{27,30,31}$.

O excesso de influxo de ácidos graxos saturados para o fígado, vindo da massa aumentada de tecido adiposo do portador de DHGNA, promove aumento de ácidos graxos saturados nas membranas celulares. Com isso, a membrana enrijece e 
diminui sua fluidez. As membranas ficam então desorganizadas e prejudicam o funcionamento das organelas. Elas acabam por se romper, promovendo a necrose celular 32,33 .

Além do influxo de gordura, a lipogênese responde pelo acúmulo de lipídeos em fígados de pacientes portadores de DHGNA. Durante uma alta disponibilidade de carboidratos, o tecido adiposo também pode converter a glicose em lipídeos, no entanto, a maior parte da síntese de ácidos graxos ocorre nos hepatócitos ${ }^{27}$.

O consumo de carboidratos de alto índice glicêmico pode favorecer naturalmente a lipogênese em situações fisiológicas em que há excedente energético. Já em pacientes com resistência à insulina a lipogênese se acentua, uma vez que a hiperinsulinemia está relacionada com o aumento da expressão de genes que promovem a lipogênese nos hepatócitos. Em uma situação pós-prandial as concentrações de glicose e insulina aumentam no plasma e ambas são requeridas para a plena ativação da lipogênese. Vale lembrar que mesmo em situações de resistência à insulina, muito frequente em portadores de DHGNA, no período pósprandial a glicose entra livremente no hepatócito, por difusão passiva, pelo transportador de glicose tipo 2 (GLUT2). Este transportador, ao contrário do GLUT4, funciona independentemente da sinalização de insulina. Desta forma, o influxo de glicose no fígado fica favorecido ${ }^{5}$ (Figura 2 ).

A frutose merece destaque por ter uma particularidade. Diferentemente da glicose, que pode ser fonte de energia para a maioria das células, a frutose é preferencialmente metabolizada no fígado e por isso é um verdadeiro combustível para a lipogênese $\mathrm{s}^{34,35,36}$. Assim como a glicose, a frutose também entra no hepatócito pelo transportador GLUT2 e, portanto, de modo independentemente da ação da insulina (Figura 2). Entretanto, em comparação com a glicose, a frutose tem uma reduzida capacidade de estimular a secreção de insulina ${ }^{37}$.

O estresse oxidativo contribui diretamente para a progressão da DHGNA. Com o acúmulo de gordura, a oxidação de lipídeos é aumentada nos hepatócitos. De fato, o fígado usa os ácidos graxos como seu principal combustível. Porém, esse aumento de oxidação é insuficiente para remover o excesso de lipídeos e evitar o acúmulo. Por outro lado, a oxidação de lipídeos aumentada promove estresse oxidativo, favorecendo a progressão da doença para um quadro inflamatório ${ }^{28}$ (Figura 2).

O balanço energético nos hepatócitos é regulado pela atividade mitocondrial. No entanto, na DHGNA ocorre uma sobrecarga de ácidos graxos livres no interior da 
mitocôndria. Isso altera a permeabilidade da membrana interna, comprometendo a capacidade de síntese de Adenosina Trifosfato (ATP) e o trânsito de prótons. Ao mesmo tempo, o aumento da oxidação de lipídeos aumenta o fluxo na cadeia transportadora de elétrons, favorecendo um extravasamento. Nesta situação os elétrons excedentes, que extravasam, reagem diretamente com o oxigênio na matriz mitocondrial, sem a participação de prótons e sem a formação de água. Desta forma, ocorre a produção exagerada do radical superóxido, que favorece uma situação de estresse oxidativo ${ }^{38}$.

O radical superóxido é considerado o principal fator da situação de toxicidade oxidante. Ele origina diferentes metabólitos de oxigênio, coletivamente chamados de espécies reativas de oxigênio (ERO), incluindo, por exemplo, o peróxido de hidrogênio e o radical hidroxila, entre outros. O acúmulo excessivo das ERO causa reações em cadeia que promovem danos às estruturas celulares. Neste cenário, as membranas celulares sofrem peroxidação lipídica, normalmente nos ácidos graxos insaturados, o que destrói suas propriedades de barreira e prejudica sua função, levando à morte celular. Além disso, $\mathrm{O}$ excesso das ERO causa danos às proteínas e ácidos nucléicos ${ }^{39,40,41}$.

A exportação de lipídeos pelo fígado está comprometida em portadores de DHGNA (Figura 2). O fígado secreta triglicerídeos por meio de VLDL-c para os tecidos periféricos, incluindo o músculo esquelético e cardíaco, além do tecido adiposo. A formação das partículas de VLDL-c e a montagem dos lipídeos com a Apolipoproteína ApoB10o ocorrem no lúmen do retículo endoplasmático (RE). A VLDL-c e a ApoB10o conectadas são requeridas para a exportação dos trigliderídeos. Cada partícula de VLDL-c é estabilizada por uma molécula de ApoB100 ${ }^{31}$.

Acúmulos moderados de lipídeos no fígado aumentam a secreção de ApoB100. No entanto, acúmulos prolongados provocam estresse e desorganização das membranas no RE, comprometendo a montagem da partícula de VLDL-c. Portanto, a exportação de lipídeos aumenta nos estágios iniciais da doença, mas diminui nos estágios subsequentes, contribuindo para a severidade do quadro ${ }^{42,43}$.

O estresse do RE está envolvido com uma significativa incapacidade de produzir proteínas com estrutura correta. O aumento de proteínas com conformação incorreta demanda excessivamente a maquinaria de reparo. Isso altera a homeostase da organela, que, desregulada, passa a ser também uma fonte produtora de ERO, contribuindo para a necrose e inflamação do fígado ${ }^{38}$. 
Figura 2 - Mecanismos do acúmulo de gordura e favorecimento do quadro inflamatório na DHGNA. A aquisição e a destinação de lipídeos são reguladas por quatro vias: aquisição de lipídeos circulantes, lipogênese, oxidação de ácidos graxos e exportação de lipídeos. O comprometimento dessas vias desenvolve a DHGNA.

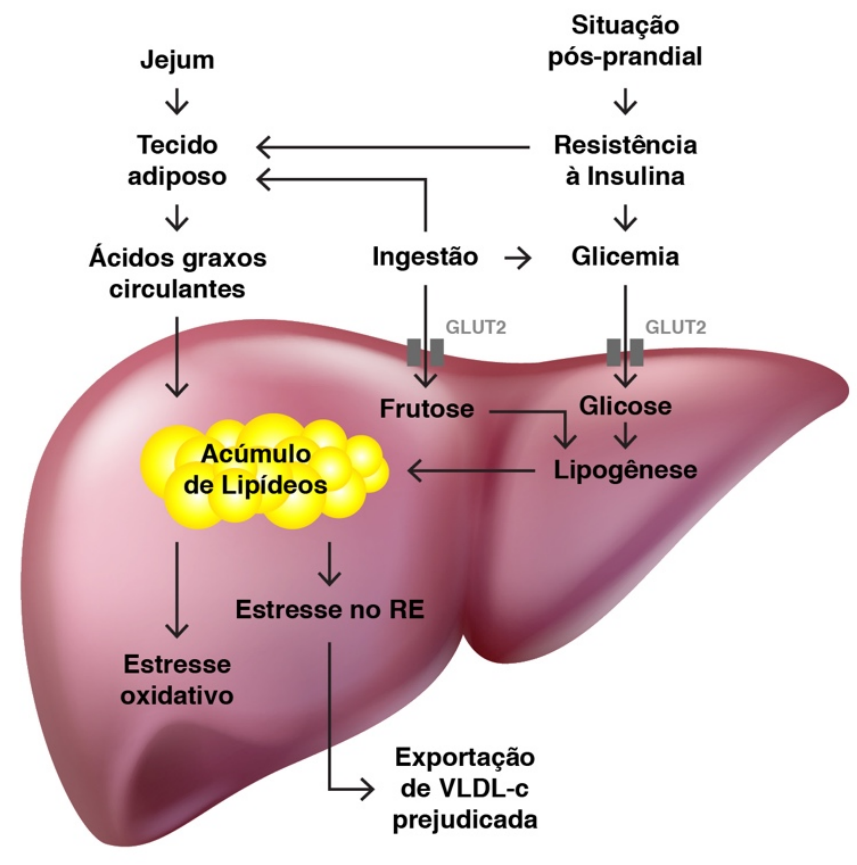

\section{Desafios terapêuticos}

Não há tratamento medicamentoso específico para a DHGNA. Apenas algumas drogas mostraram certa eficácia em ensaios clínicos randomizados, porém, os efeitos adversos apresentados inviabilizaram o potencial de uso no tratamento ${ }^{44,45}$. De fato, não é trivial o desenvolvimento e a disponibilização de uma droga para a DHGNA. Os mecanismos moleculares envolvidos, relacionados com o acúmulo de lipídeos no fígado e suas consequências, são complexos e estritamente interconectados. É comum haver reguladores que exercem mais de uma função em variados contextos fisiológicos. Por isso, qualquer intervenção voltada a um ou mais componentes, de uma determinada via, pode ter consequências em múltiplas vias de sinalização celular. Outro complicador é o fato de haver diferenças entre os indivíduos. Tudo isso deve ser levado em conta para superar o desafio do desenvolvimento de futuras opções de tratamento farmacológico ${ }^{28}$. Nesse contexto, as recomendações atuais para o tratamento da DHGNA são as modificações no estilo de vida. No entanto, intervenções no estilo de vida são notadamente difíceis de serem aceitas e mantidas. Por isso, há o apelo social pelo desenvolvimento de uma terapia farmacológica com a qual os pacientes poderiam se beneficiar ${ }^{46}$. 
O aporte calórico excessivo, os maus hábitos alimentares e o sedentarismo são os principais causadores da DHGNA. No entanto, os profissionais de saúde não devem deixar de investigar as outras causas menos frequentes, entre elas os medicamentos hepatotóxicos, os esteroides anabolizantes e exposição às aflatoxinas ${ }^{19,20,21}$.

A ausência de tratamento farmacológico específico coloca obrigatoriamente as modificações do estilo de vida no foco do tratamento. As recomendações envolvem a adoção de hábitos alimentares saudáveis associados à prática regular de exercícios físicos e perda de peso. No entanto, os componentes culturais, ambientais, genéticos, dietéticos, físicos e psicológicos, dos pacientes, em diferentes combinações, dificultam as mudanças de hábitos necessárias. A distância entre o discurso do profissional nutricionista e a prática das mudanças de hábitos pode ser grande. E quanto maior essa distância, maiores são as frustrações do paciente e do profissional. Antigos hábitos alimentares duradouros e consolidados são de fato difíceis de serem substituídos ${ }^{17}$.

O desafio se torna ainda maior nos casos em que a DHGNA ainda está na fase de esteatose não inflamatória, e por isso, assintomática e sem maiores consequências em curto prazo. Para transformar os hábitos alimentares, as mudanças devem ser propostas de modo gradual e sem restrições exageradas. Além disso, o plano alimentar deve ser flexível, do contrário, o indivíduo adere às novas práticas por algum período, podendo perder peso e melhorar o quadro. Mas depois, se vendo muito distante dos hábitos antigos e consolidados, abandona o plano, regride o quadro e se frustra. Deste modo, deve ser dada atenção especial à relação do paciente com o ato de se alimentar, para a obtenção de resultados duradouros. Nesse contexto, uma abordagem multiprofissional é indicada para a obtenção de melhores resultados. o acompanhamento psicológico é importante e o profissional nutricionista pode orientar o paciente a buscar a ajuda de um profissional de psicologia. $O$ nutricionista pode também estimular o paciente a buscar a orientação de um profissional de educação física, para associar a prática de exercícios físicos aos novos hábitos alimentares ${ }^{47}$.

Os hábitos alimentares sofrem importante influência das situações emocionais vividas no cotidiano. Existem diferenças importantes entre as chamadas: fome fisiológica e a fome emocional. Um profissional de psicologia, com conhecimento na área, pode ajudar a entender melhor as emoções que interferem na alimentação, oferecendo alternativas para lidar com os desejos alimentares não fisiológicos ${ }^{48}$. 
São diversos os benefícios proporcionados pelos exercícios físicos. Existem evidências de redução de gordura no fígado proporcionada pela prática regular de exercícios aeróbicos ${ }^{49}$. A prática de musculação moderada, com foco em exercícios de força, também traz benefícios, podendo reduzir a gordura e a inflamação do fígado, antes mesmo de haver perda de peso significativa ${ }^{50}$. Por outro lado, a prática de exercícios exagerada, que leva à exaustão e ao sobretreinamento, pode piorar o quadro e até induzir o acúmulo de gordura no fígado ${ }^{51}$.

\section{Terapia nutricional}

A melhora do quadro de DHGNA passa obrigatoriamente pela diminuição da massa de tecido adiposo. No entanto, vale destacar que restrições calóricas drásticas, apesar de levarem à perda de peso rápida, não são recomendadas por conta do consequente aumento da concentração de ácidos graxos circulantes, mobilizados do tecido adiposo, que podem aumentar o influxo de ácidos graxos no fígado agravando o quadro ${ }^{52,53}$. Por outro lado, a diminuição calórica e a consequente perda de peso são fatores chave para a redução do acúmulo de gordura no fígado. Estudos apontam que, em grande parte dos casos, uma perda de peso de 7 a $10 \%$ já pode reduzir a gordura do fígado e inclusive reduzir a fibrose, nos casos mais avançados da DHGNA $^{54,55}$.

O plano alimentar, tanto para prevenção como para tratamento da DHGNA, deve levar em consideração algumas observações importantes. Uma alta ingestão de gorduras aumenta o risco de DHGNA e agrava o quadro clínico. Entretanto, a ingestão exagerada de ácidos graxos saturados aumenta mais significativamente o acúmulo de gordura no fígado, em comparação com a ingestão exagerada de ácidos graxos insaturados ${ }^{56}$.

A ingestão de gordura trans também contribui para a esteatose hepática ${ }^{57,58}$. Ao mesmo tempo, dietas ricas em ácidos graxos monoinsaturados e poliinsaturados tendem a reduzir a gordura do fígado ${ }^{59}$. Além disso, existem evidências de comprometimento da função das membranas celulares, por conta do influxo celular de ácidos graxos saturados e trans. Nesta situação as membranas se tornam rígidas e perdem a flexibilidade. Por outro lado, o influxo de ácidos graxos insaturados promove a flexibilidade e a funcionalidade das membranas celulares. Vale lembrar que danos às membranas celulares contribuem para o quadro inflamatório da DHGNA $^{60,61}$. Por isso, a recomendação é substituir ao máximo o consumo de ácidos graxos saturados e trans por ácidos graxos mono e poliinsaturados ${ }^{62}$. 
O consumo de carboidratos de alto índice glicêmico deve ser controlado por favorecer a lipogênese, especialmente em portadores de diabetes tipo $2^{5}$. Além da glicose, a alta ingestão de frutose tem grande importância no favorecimento do acúmulo de ácido graxo no hepatócito. Ambas respondem pelo potencial de desenvolvimento de DHGNA, por conta do excesso de açúcares simples na dieta. Portanto, produtos processados, contendo sacarose e xarope de milho, particularmente com altas concentrações de frutose, devem ser evitados por favorecerem significativamente a lipogênese hepática ${ }^{34,35,36}$.

O consumo de frutose representa uma questão de saúde pública e por isso merece atenção redobrada. $\mathrm{O}$ alto consumo de guloseimas e bebidas doces, ricos em frutose, está ligado à ocorrência de síndrome metabólica. Nos últimos 40 anos tem havido um crescimento significativo do consumo de frutose. Esse consumo crescente vem acontecendo junto com o crescimento da incidência e da prevalência de obesidade e síndrome metabólica. Além disso, por ser menos capaz de produzir saciedade e por ser mais palatável, a frutose estimula um alto consumo ${ }^{63}$.

A dieta deve ser normoglicídica e normolipídica, atendendo ao requerimento energético, voltada para o estado nutricional de eutrofia e com atenção à composição de carboidratos e lipídeos ${ }^{64}$. Apesar de ser recomendado que a dieta tenha baixo teor de carboidratos simples, não há necessidade de restrição absoluta. Vale lembrar que não são a glicose e a frutose em si, os únicos fatores de risco para esteatose, mas também o excesso de calorias ingeridas e o excesso de peso ${ }^{20,65}$. Nesse contexto, a diminuição calórica na dieta deve prever uma restrição de açúcares simples, ácidos graxos saturados e ácidos graxos trans. O controle do aporte calórico da dieta pode ser mais voltado para a diminuição de gordura ou de carboidratos, desde que a restrição calórica seja mantida e que a proporção entre os macronutrientes e o aporte de micronutrientes seja respeitada ${ }^{64,66}$.

Determinados compostos bioativos com potenciais efeitos benéficos podem ajudar a prevenir a DHGNA e também auxiliar no tratamento. Esses compostos podem modular os mecanismos moleculares que levam ao acúmulo de gordura, estresse oxidativo, inflamação e fibrose hepática. Desta forma, os pacientes podem se beneficiar com a ingestão de alimentos ricos em ômega 3, vitamina E, vitamina $D$, polifenóis, flavonoides, resveratrol, cobre, selênio e ferro. Esses compostos possuem funções variadas, entre elas algumas específicas e outras em comum. Em conjunto esses nutrientes podem, por um lado, auxiliar a reduzir: a esteatose 
hepática, a lipogênese, a inflamação hepática, a gordura visceral, a fibrose hepática e o estresse oxidativo; e por outro lado, auxiliar a aumentar a atividade antioxidante, a lipólise, a beta-oxidação, o nível das enzimas desintoxicantes e antioxidantes do fígado e o metabolismo de glicose ${ }^{17}$.

Por último, mas não menos importante, o consumo excessivo de bebida alcoólica (mais do que $20 \mathrm{~g} /$ dia) deve ser evitado, em pacientes com esteatose hepática confirmada, para não favorecer o acúmulo de gordura no fígado. Já em pacientes com um quadro mais avançado, em que haja inflamação e fibrose hepática, o consumo de bebida alcoólica deve ser evitado, pela possibilidade de acelerar o agravamento do quadro para carcinoma hepatocelular ${ }^{62}$.

\section{CONSIDERAÇÕES FINAIS}

As informações aqui reunidas refletem o apontamento da literatura científica sobre a importância da DHGNA e sua relação com o excesso de peso, a diabetes tipo 2, a dislipidemia, a resistência à insulina e o risco cardiovascular. É reportado também as questões que envolvem a inexistência de um tratamento medicamentoso específico para a DHGNA e a recomendação da terapia nutricional. Nesse contexto, foram sintetizados conceitos e conhecimentos que ajudam a esclarecer os fenômenos metabólicos e os efeitos dos alimentos envolvidos na DHGNA, apontando possibilidades para as abordagens de terapia e educação alimentar e nutricional, para a prevenção e o tratamento da doença. Com isso, foram disponibilizadas informações que ambientam os profissionais de saúde, em especial os profissionais nutricionistas, no cenário atual dos temas que envolvem a DHGNA.

\section{REFERÊNCIAS}

1. Byass P. The global burden of liver disease: a challenge for methods and for public health. BMC Med. 2014; 12:159.

2. Oliveira CPMS, Mazo DFC. Hepatologia/Gastroenterologia - Doença hepática gordurosa não alcoólica: fisiopatogenia e relação com a terapêutica. In: Parise ER, Mendonça Filho HTF. Esteatose hepática: visão hepatológica e cardiometabólica. 1. ed. São Paulo: MTS Editora Arte de Cuidar; 2013.

3. Sayiner M, Koenig A, Henry L, Younossi ZM. Epidemiology of nonalcoholic fatty liver disease and nonalcoholic steatohepatitis in the United States and the rest of the world. Clin Liver Dis. 2016;20(2):205-14.

4. Bellentani S. The epidemiology of non-alcoholic fatty liver disease. Liver Int. 2017;37(1):81-4. 
5. Hardy T, Oakley F, Anstee QM, Day CP. Nonalcoholic Fatty Liver Disease: Pathogenesis and Disease Spectrum. Annu Rev Pathol: Mechanisms of Disease. 2016; 11:451-96.

6. Parise ER, Santos VN. Hepatologia/Gastroenterologia. Esteatose: definição, epidemiologia e diagnóstico. In: Parise ER, Mendonça Filho HTF. Esteatose hepática: visão hepatológica e cardiometabólica. 1. ed. São Paulo: MTS Editora Arte de Cuidar; 2013.

7. Parise ER, Mendonça Filho HTF. Esteatose hepática: visão hepatológica e cardiometabólica. 1. ed. São Paulo: MTS Editora Arte de Cuidar; 2013.

8. Ertle J, Dechêne A, Sowa JP, Penndorf V, Herzer K, Kaiser G, Schlaak JF, Gerken G, Syn WK, Canbay A. Non-alcoholic fatty liver disease progresses to hepatocellular carcinoma in the absence of apparent cirrhosis. Int J Cancer. 2011;128(10):2436-43.

9. Pockros PJ, Bisceglie AMD, Bloom A. Direct-acting antivirals for the treatment of hepatitis C virus infection. [Internet]. UpToDate. [acesso em 5 out 2018] Disponível em: https://www.uptodate.com/contents/direct-acting-antiviralsfor-the-treatment-of-hepatitis-c-virus-infection/print.

10. Younossi Z, Stepanova M, ONG JP, Jacobson IM, Bugianesi E, Duseja A, Eguchi Y, Wong VW, Negro F, Yilmaz Y, Romero-Gomez M, George J, Ahmed A, Wong R, Younossi I, Ziayee M, Afendy A. Global nonalcoholic steatohepatitis council. Nonalcoholic steatohepatitis is the fastest growing cause of hepatocellular carcinoma in liver transplant candidates. Clin Gastroenterol Hepatol. 2018;18:30611-6.

11. Bertot LC, Adams LA. The natural course of non-alcoholic fatty liver disease. Int J Mol Sci. 2016;17(5):774.

12. Asselah T, Boyer N, Guimont MC, Cazals-Hatem D, Tubach F, Nahon K, Daïkha H, Vidaud D, Martinot M, Vidaud M, Degott C, Valla D, Marcellin P. Liver fibrosis is not associated with steatosis but with necroinflammation in French patients with chronic hepatitis C. Gut. 2003; 52:1638-43.

13. Neuman MG, Schmilovitz-Weiss H, Hilzenrat N, Bourliere M, Marcellin $P$, Trepo C, Mazulli T, Moussa G, Patel A, Baig AA, Cohen L. Markers of inflammation and fibrosis in alcoholic hepatitis and viral hepatitis C. Int J Hepatology. 2012;2012:231210.

14. Marcellin P, Gane E, Buti M, Afdhal N, Sievert W, Jacobson IM, Washington MK, Germanidis G, Flaherty JF, Aguilar Schall R, Bornstein JD, Kitrinos KM, Subramanian GM, Mchutchison JG, Heathcote EJ. Regression of cirrhosis during treatment with tenofovir disoproxil fumarate for chronic hepatitis B: a 5-year open-label follow-up study. Lancet. 2013;381(9865):468-75.

15. Marcellin P, Kutala BK. Liver diseases: a major, neglected global public health problem requiring urgent actions and large-scale screening. Liver Int. 2018;38(1):2-6.

16. Dyson JK, Anstee QM, Mcpherson S. Non-alcoholic fatty liver disease: a practical approach to diagnosis and staging. Frontline Gastroenterol. 2014;5:211-218.

17. Dongiovanni P, Lanti C, Riso P, Valenti L. Nutritional therapy for nonalcoholic fatty liver disease. J Nutr Biochem. 2016;29:1-11. 
18. Biblioteca virtual em saúde. [Internet]. Descritores em ciências da saúde. [acesso em 13 set 2018]. Disponível em: http://decs.bvs.br.

19. Amaya-Farfan J. Aflatoxin B1-induced hepatic steatosis: role of carbonyl compounds and active diols on steatogenesis. Lancet. 1999; 353(9154):747-8.

20. Sociedade brasileira de hepatologia. [Internet]. Doença hepática gordurosa não alcoólica. [acesso em 3 out 2018]. Disponível em: http://www.sbhepatologia.org.br/pdf/Consenso_DHGNA_da_SBH-2015.pdf.

21. Miele L, Liguori A, Marrone G, Biolato M, Araneo C, Vaccaro FG, Gasbarrini A, Grieco A. Fatty liver and drugs: the two sides of the same coin. Eur Rev Med Pharmacol Sci. 2017;21(1):86-94.

22. Kumar R, Mohan S. Non-alcoholic fatty liver disease in lean subjects: characteristics and implications. J Clin Transl Hepatol. 2017;5(3):216-23.

23. Curi R, Pompéia C, Miyasaka CK, Procopio J. Entendendo a gordura: os ácidos graxos. 1a ed. Barueri - SP: Editora Manole; 2002.

24. Manuais MSD. [Internet]. Versão saúde para a família. [acesso em 5 out 2018]. Disponível em: https://www.msdmanuals.com/pt-br/casa/doenças-hepáticase-da-ves\%C3\%ADcula-biliar/fibrose-e-cirrose-do-f\%C3\%ADgado/fibrosedo-f\%C3\%ADgado.

25. Parise ER. Métodos de imagem e testes não invasivos no diagnóstico da DHGNA. Doença hepática gordurosa não alcoólica. [Internet]. Sociedade brasileira de hepatologia. [acesso em 3 out 2018]. Disponível em: http://www.sbhepatologia.org.br/pdf/revista_monotematico_hepato.pdf.

26. BRASIL. Ministério da Saúde. [Internet]. Elastografia hepática ultrassônica no diagnóstico da fibrose hepática. Comissão nacional de incorporação de tecnologias no SUS. [acesso em 3 out 2018]. Disponível em: http://conitec.gov.br/images/Relatorios/2015/Elastografia_final.pdf.

27. Nelson DL, Cox MM. Regulação hormonal e integração do metabolismo em mamíferos. In: Princípios e Bioquímica de Lehninger. 6. ed. Porto Alegre - RS: Artmed; 2014.

28. Ipsen DH, Lykkesfeldt J, Tveden-Nyborg P. Molecular mechanisms of hepatic lipid accumulation in non-alcoholic fatty liver disease. Cell Mol Life Sci. 2018;75:3313-27.

29. Bechmann LS, Hannivoort RA, Gerken G, Hotamisligil GS, Trauner M, Canbay A. The interaction of hepatic lipid and glucose metabolism in liver diseases. J Hepatol. 2012;56:952-64.

30. Projeto diretrizes [Internet]. Associação médica brasileira e conselho federal de medicina. Terapia nutricional nas doenças hepáticas crônicas e insuficiência hepática. [acesso em 3 out 2018]. Disponível em: https://diretrizes.amb.org.br/_BibliotecaAntiga/terapia_nutricional_nas_doe ncas_hepaticas_cronicas_e_insuficiencia_hepatica.pdf.

31. Kawano Y, Cohen DE. Mechanisms of hepatic triglyceride accumulation in nonalcoholic fatty liver disease. J Gastroenterol. 2013;48(8):434-41.

32. Zámbó V, Simon-Szabó L, Szelényi P, Kereszturi E, Bánhegyi G, Csala M. Lipotoxicity in the liver. World J Hepatol. 2013;5(10):550-7. 
33. Weijers RNM. Membrane flexibility, free fatty acids, and the onset of vascular and neurological lesions in type 2 diabetes. J Diabetes Metab Disord. 2016;15(13).

34. Sobrecases H, Lê KA, Bortolotti M, Schneiter P, Ith M, Kreis R, Boesch C, Tappy L. Effects of short-term overfeeding with fructose, fat and fructose plus fat on plasma and hepatic lipids in healthy men. Diabetes Metab. 2010;36(3):244-6.

35. Jensen T, Abdelmalek MF, Sullivan S, Nadeau KJ, Green M, Roncal C, Nakagawa T, Kuwabara M, Sato Y, Kang DH, Tolan DR, Sanchez-Lozada LG, Rosen HR, Lanaspa MA, Diehl AM, Johnson RJ. Fructose and Sugar: A Major Mediator of Nonalcoholic Fatty Liver Disease. J Hepatol. 2018;68(5):1063-75.

36. Cuppari L. Nutrição clínica no adulto. 4a ed. Barueri - SP: Editora Manole; 2019.

37. Samuel VT. Fructose induced lipogenesis: from sugar to fat to insulin resistance. Trends Endocronol Metab. 2011;22(2):60-5.

38. Masarone M, Rosato V, Dallio M, Gravina AG, Aglitti A, Loguercio C, Federico A, Persico M. Role of Oxidative Stress in Pathophysiology of Nonalcoholic Fatty Liver Disease. Oxid Med Cell Longev. 2018;2018:1-14.

39. Bergamini CM, Gambetti S, Dondi A, Cervellati C. Oxygen, reactive oxygen species and tissue damage. Curr Pharm Des. 2004;10(14): 1611-26.

40. Stark G. Functional consequences of oxidative membrane damage. J Membr Biol. 2005;205(1):1-16.

41. Indo HP, Yen HC, Nakanishi I, Matsumoto K, Tamura M, Nagano Y, Matsui H, Gusev O, Cornette R, Okuda T, Minamiyama Y, Ichikawa H, Suenaga S, Oki M, Sato T, Ozawa T, Clair DK, Majima HJ. A mitochondrial superoxide theory for oxidative stress diseases and aging. J Clin Biochem Nutr. 2015;56(1):1-7.

42. Ota T, Gayet C, Ginsberg HN. Inhibition of apolipoprotein B100 secretion by lipid-induced hepatic endoplasmic reticulum stress in rodents. J Clin Invest. 2008;118:316-32.

43. Zhang XQ, Xu CF, Yu CH, Chen WX, Li YM. Role of endoplasmic reticulum stress in the pathogenesis of nonalcoholic fatty liver disease. World J Gastroenterol. 2014;20:1768-76.

44. Sanyal AJ, Chalasani N, Kowdley KV, Mccullough A, Diehl AM, Bass NM, Neuschwander-Tetri BA, Lavine JE, Tonascia J, Unalp A, Van Natta M, Clark J, Brunt EM, Kleiner DE, Hoofnagle JH, Robuck PR. Pioglitazone, vitamin E, or placebo for nonalcoholic steatohepatitis. N Engl J Med. 2010;362:1675-85.

45. Neuschwander-Tetri BA, Loomba R, Sanyal AJ, Lavine JE, Van Natta ML, Abdelmalek MF, Chalasani N, Dasarathy S, Diehl AM, Hameed B, Kowdley KV, Mccullough A, Terrault N, Clark JM, Tonascia J, Brunt EM, Kleiner DE, Doo E. Farnesoid X nuclear receptor ligand obeticholic acid for non-cirrhotic, nonalcoholic steatohepatitis (FLINT): a multicentre, randomised, placebocontrolled trial. Lancet. 2015;385:956-65.

46. Middleton KR, Anton SD, Perri MG. Long-term adherence to health behavior change. Am J Lifestyle Med. 2013;7:395-404.

47. Galizzi Filho J. Mudanças no estilo de vida. Doença hepática gordurosa não alcoólica [Internet]. Sociedade brasileira de hepatologia. [acesso em 3 out 2018]. Disponível em:

http://www.sbhepatologia.org.br/pdf/revista_monotematico_hepato.pdf. 
48. Help Guide. [Internet]. Emotional eating. How to recognize and stop emotional and stress eating. [acesso em 11 mai 2019]. Disponível em:

https://www.helpguide.org/articles/diets/emotional-eating.htm/.

49. Muñoz VR, Gaspar RC, Kuga GK, Nakandakari SCBR, Baptista IL, Mekary RA, da Silva ASR, de Moura LP, Ropelle ER, Cintra DE, Pauli JR. Exercise decreases CLK2 in the liver of obese mice and prevents hepatic fat accumulation. J Cell Biochem. 2018;119(7):5885-5892.

50. Pereira RM, Rodrigues KCDC, Anaruma CP, Sant'Ana MR, de Campos TDP, Gaspar RS, Canciglieri RDS, de Melo DG, Mekary RA, da Silva ASR, Cintra DE, Ropelle ER, Pauli JR, de Moura LP. Short-term strength training reduces gluconeogenesis and NAFLD in obese mice. J Endocrinol. 2019;241(1):59-70.

51. Da Rocha AL, Pinto AP, Teixeira GR, Pereira BC, Oliveira LC, Silva AC, Morais GP, Cintra DE, Pauli JR, da Silva ASR. Exhaustive Training Leads to Hepatic Fat Accumulation. J Cell Physiol. 2017;232(8):2094-2103.

52. Kneeman JM, Misdraji J, Corey KE. Secondary causes of nonalcoholic fatty liver disease. Therap Adv Gastroenterol. 2012;5(3):199-207.

53. Hospital Albert Einstein. Esteatose hepática. [Internet]. Guia de doenças e sintomas. [acesso em 3 out 2018]. Disponível em:

https://www.einstein.br/Pages/Doenca.aspx?eid=251.

54. Marchesini G, Mazzotti A. NAFLD incidence and remission: only a matter of weight gain and weight loss? J Hepatol. 2015;62(1):15-7.

55. Marchesini G, Petta S, Dalle Grave R. Diet, weight loss, and liver health in nonalcoholic fatty liver disease: Pathophysiology, evidence, and practice. Hepatology. 2016;63(6):2032-43.

56. Green CJ, Hodson L. The influence of dietary fat on liver fat accumulation. Nutrients. 2014;6(11):5018-33.

57. Machado RM, Stefano JT, Oliveira CP, Mello ES, Ferreira FD, Nunes VS, De lima VM, Quintão EC, Catanozi S, Nakandakare ER, Lottenberg AM. Intake of trans fatty acids causes nonalcoholic steatohepatitis and reduces adipose tissue fat content. J Nutr. 2010;140(6):1127-32.

58. Dhibi M, Brahmi F, Mnari A, Houas Z, Chargui I, Bchir L, Gazzah N, Alsaif MA, Hammami $M$. The intake of high fat diet with different trans fatty acid levels differentially induces oxidative stress and non alcoholic fatty liver disease (NAFLD) in rats. Nutr Metab. 2011;8(1):65.

59. Rosqvist F, Iggman D, Kullberg J, Cedernaes J, Johansson HE, Larsson A, Johansson L, Ahlström H, Arner P, Dahlman I, Risérus U. Overfeeding polyunsaturated and saturated fat causes distinct effects on liver and visceral fat accumulation in humans. Diabetes. 2014;63(7):2356-68.

60. Escribá PV. Membrane-lipid therapy: A historical perspective of membranetargetedtherapies - From lipid bilayer structure to the pathophysiologicalregulation of cells. Biochim Biophys Acta Biomembr. 2017;1859(9):1493-506.

61. Shen Y, Zhao Z, Zhang L, Shi L, Shahriar S, Chan RB, Di Paolo G, Min W. Metabolic activity induces membrane phase separation in endoplasmic reticulum. Proc Natl Acad Sci USA. 2017;114(51):13394-99. 
62. George ES, Forsyth A, Itsiopoulos C, Nicoll AJ, Ryan M, Sood S, Roberts SK, Tierney AC. Practical Dietary Recommendations for the Prevention and Management of Nonalcoholic Fatty Liver Disease in Adults. Adv Nutr. 2018;9(1):30-40.

63. Pereira RM, Botezelli JD, da Cruz Rodrigues KC, Mekary RA, Cintra DE, Pauli JR, da Silva ASR, Ropelle ER, de Moura LP. Fructose Consumption in the Development of Obesity and the Effects of Different Protocols of Physical Exercise on the Hepatic Metabolism. Nutrients. 2017 Apr 20;9(4):405.

64. Parry SA, Hodson L. Influence of dietary macronutrients on liver fat accumulation and metabolismo. J Investig Med. 2017;65(8):1102-15.

65. Wehmeyer MH, Zyriax BC, Jagemann B, Roth E, Windler E, Schulze Zur Wiesch J, Lohse AW, Kluwe J. Nonalcoholic fatty liver disease is associated with excessive calorie intake rather than a distinctive dietary pattern. Medicine (Baltimore). 2016;95(23):e3887.

66. Haufe S, Engeli S, Kast P, Böhnke J, Utz W, Haas V, Hermsdorf M, Mähler A, Wiesner S, Birkenfeld AL, Sell H, Otto C, Mehling H, Luft FC, Eckel J, SchulzMenger J, Boschmann M, Jordan J. Randomized comparison of reduced fat and reduced carbohydrate hypocaloric diets on intrahepatic fat in overweight and obese human subjects. Hepatology. 2011;53(5):1504-14.

Submissão: $29 / 06 / 2020$

Aprovação: 02/12/2020 